\title{
Nonlinear mixed-state longitudinal and transverse resistivities of superconductors with anisotropic pinning - a phenomenological approach
}

\author{
Valerij A. Shklovskij \\ National Science Center «Kharkov Institute of Physics and Technology», Institute of Theoretical Physics,
} 1 Akademicheskaya St., 310108, Kharkov, Ukraine

Kharkov State University, Physical Department, 4 Svobody Sq., 310077, Kharkov, Ukraine

E-mail: valerij.a.shklovskij@univer.kharkov.ua

Received September 24, 1998

\begin{abstract}
In the presence of anisotropic pinning due to unidirected twins, the nonlinear vortex dynamics is discussed in terms of phenomenologically introduced anisotropic drag and pinning viscosities. The theoretical basis for experimental reconstruction of these viscosities is proposed. The nonlinear Ohm's law is derived. Assuming the anisotropic pinning alone ( $a$-pinning model): a) new scaling relations for the anisotropic Hall conductivity are predicted; b) nonlinear guiding effects are discussed; c) specific current and angular behavior of current-voltage characteristics are analyzed.
\end{abstract}

PACS: $74.60 . \mathrm{Ge}, 74.60 . \mathrm{Jg}$

The influence of twin boundaries (TB's) on the transport properties of high- $T_{c}$ superconductors is a topic of great current interest [1-11,16-18]. One of the reasons for this interest is that the TB's are naturally occurring planar defects that can easily be formed in a high- $T_{c} \mathrm{YBa}_{2} \mathrm{Cu}_{3} \mathrm{O}_{7-\delta}(\mathrm{YBCO})$ compound.

It is generally recognized that the order parameter is slightly suppressed at TB's [1]. As result, an isolated TB attracts vortices and pins them [1]. The $\mathrm{TB}$ pinning force acting on the vortices directed along the $\mathbf{c}$-axis (and the TB) of the crystal is often strongly anisotropic, because it is usually considerably weaker for the motion of vortices along twins than across them [2].

Recently, the problem of twin influence on the vortex motion in plane geometry has been studied numerically $[3,4]$. The simulations in $[3,4]$ were performed for the interaction of moving vortices with only one isolated TB. Some interesting dynamic peculiarities of this interaction were elucidated. However, it is worth noting that, in a real transport experiment $[2,5-10]$ we usually probe a certain «self-averaged» vortex dynamics, which results from the interaction of vortices with many TB's, distributed with some average density between voltage leads. Obviously, this self-averaging will «smear» some subtle details of the vortex interaction with an isolated $\mathrm{TB}$, which were detected in $[3,4]$.

Several Hall experiments [5-7] were performed on YBCO samples, where TB's were oriented basically in two mutually orthogonal directions. Because the transport response of the crystals was always measured as an integral property, the pinning anisotropy of twins in this case was commonly masked, i.e., the influence of TB's pinning on $a b$-plane transport in the $\mathbf{H} \| \mathbf{c}$ geometry is, on the average, isotropic, as for point pins (neglecting small $a b$-axis anisotropy).

A much different type of situation (anisotropic) occurs if we measure the $a b$-plane transport response of single crystal with unidirected twins $[2,8-10]$. It is generally believed [11] that the main special feature of this response lies in the possibility of the «guided» motion (GM) of vortices along the easiest direction (in the case of TB's mostly along them). This GM generates a new, specific contribution to the transverse (with respect to the current direction) resistivity of the sample $\rho_{\perp}^{+}$, which is even with respect to the magnetic field reversal (in addition to the odd Hall contribution $\rho_{\perp}^{-}$, inherent in the isotropic pinning contribution). 
Earlier, experimental and certain theoretical aspects of anisotropic pinning and GM of vortices moving in the flux flow regime (for cold-rolled $\mathrm{Nb}-\mathrm{Ta}$ sheets) have been discussed in detail by Niessen and Weijesenfeld in [12]. Interest in these problems was renewed after detection of TB's in YBCO. Apart from the experimental works [2,5-10], we should also mention in this connection the recent theoretical paper by Mawatari [11], where the single-vortex anisotropic pinning dynamics has been discussed within the frame-work of the «microscopic» approach based on the Fokker-Planck equation.

Another approach to the anisotropic pinning was first suggested by Sonin and Kholkin in [13]. They proposed the general form of a linear Ohm's law in uniaxially anisotropic media, which was formulated (on the basis of symmetry considerations) in terms of four phenomenologically introduced «intrinsic» resistivities [13]. In this approach, besides $\rho_{\perp}^{+}$(and in addition to the usually measured even longitudunal contribution $\rho_{\|}^{+}$), a new, angle-dependent, odd longitudinal resistivity $\rho_{\|}^{-}$also appears, due to a possible anisotropy of the Hall drag coefficient in the twinned sample. The last effect has recently been observed for the first time in a YBCO single crystal with unidirected twins [9].

Note, however, that there are no reasons to expect the change of the Hall drag coefficient $\alpha_{i}$ due to point pins, for isotropic pinning [14,15]. This directly implies a simple scaling relation $\rho_{\perp}^{-} \sim \alpha_{i}\left(\rho_{\|}^{+}\right)^{2}$ between current-dependent, nonlinear resistivities $\rho_{\perp}^{-}(j)$ and $\rho_{\|}^{+}(j)$ for $\rho_{\perp}^{-} \ll \rho_{\|}^{+}[14,15]$.

In order to study possible scaling relations within the frame-work of the phenomenological approach [13], we have generalized its results to the nonlinear case. In so doing, we follow the phenomenological approach used recently by Vinokur et al. in [14] for the case of isotropic pinning. Below we use the method of [14] for considering both the isotropic and anisotropic pinning, and so we can derive the nonlinear Ohm's law, which was postulated earlier for the linear case in [13]. In this way we clarify the origin of the earlier introduced in [13] four phenomenological resistivities in terms of drag and pinning viscosities, i.e., at a more detailed level. Below we also show that these viscosities can be reconstructed (for purely anisotropic pinning) from current-voltage measurements in two simple special experimental LT-geometries (see Figs. 1,b,c).

The main advantage of this phenomenological approach (for example, in comparison with the microscopic approach in [11]) lies in the possibility

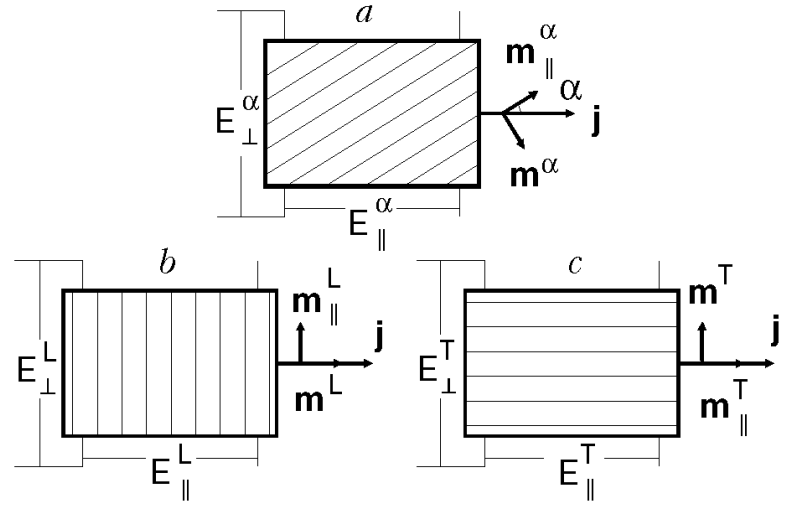

Fig. 1. The schematic sample configuration for three cases with different values of angle $\alpha$ between the current density vector $\mathbf{j}$ and the unit vector $\mathbf{m}_{\|}$directed along TB's, which are shown by thin parallel lines: general case, $\alpha \neq 0, \pi / 2(a)$; longitudinal L-geometry, $\mathbf{j} \perp \mathbf{m}_{\|}^{L}, \alpha=\pi / 2(b)$; transverse T-geometry, $\mathbf{j} \perp \mathbf{m}_{\|}^{T}, \alpha=0(c)$; in all cases $E_{\perp}$ and $E_{||}$are transverse and longitudinal (with respect to $\mathbf{j}$-direction) electric-field components, $\mathbf{m} \perp \mathbf{m}_{\|}$

of predicting and explaining the most general aspects of self-averaged vortex motion in the presence of TB's anisotropy in simple, physically transparent terms. We can than elucidate the appearance of two new anisotropic contributions to longitudinal and transverse resistivities (odd $\rho_{\|}^{-}$and even $\rho_{\perp}^{+}$, respectively), the scaling of anisotropic Hall conductivities, the nontrivial angular dependence of currentvoltage characteristic's (CVC's) in a nonlinear regime, the peculiarities of nonlinear guiding of the vortices, and some other general results.

a) Discussion of the model and nonlinear Ohm's law derivation. To be specific, let us consider the YBCO single crystal with unidirected twins in the geometry, where a homogeneous transport current of density $\mathbf{j}$ flows in the $a b$-plane and external magnetic field $\mathbf{H}$ is directed along the $\mathbf{c}$-axis. We ignore below small $a b$-plane anisotropy of the detwinned crystal; so, all the anisotropic effects under consideration are caused by TB's with the average distance between them $d>>a_{0}$, where $a_{0}$ is the intervortex distance. In this limit we can suppose that $\varepsilon \equiv a_{0} / d$ is the relative fraction of vortices trapped by the twins. The TB's presence changes both electronic and pinning properties of the previously isotropic crystal. Let $\eta_{i}$ and $\alpha_{i}$ be isotropic (bulk) vortex drag and Hall drag viscosities, respectively. In addition, we attribute the anisotropy of these viscosities to the vortices being at the TB's. Then for appropriate viscous drag and Magnus forces we have 


$$
\begin{aligned}
& \mathbf{f}_{\eta}^{i}=-\eta_{i} \mathbf{v}, \quad \mathbf{f}_{\eta}^{a}=-\eta_{i} \mathbf{v}-\eta_{t} \mathbf{v}_{t}-\eta_{l} \mathbf{v}_{l}, \\
& \mathbf{f}_{M}^{i}=-\alpha_{i} \mathbf{v} \times \mathbf{n}, \\
& \mathbf{f}_{M}^{a}=-\alpha_{i} \mathbf{v} \times \mathbf{n}-\alpha_{t} \mathbf{v}_{t} \times \mathbf{n}-\alpha_{l} \mathbf{v}_{l} \times \mathbf{n} .
\end{aligned}
$$

Here $\mathbf{v}_{t}$ and $\mathbf{v}_{l}$ are transverse and longitudinal (with respect to $\mathrm{TB}$ ) average vortex velocities, respectively $\left(\mathbf{v}=\mathbf{v}_{l}+\mathbf{v}_{t}\right.$, see Fig. 2$) ; \eta_{t}, \eta_{l}$ and $\alpha_{t}, \alpha_{l}$ are the corresponding excess anisotropic viscosities (as compared to isotropic contributions); $\mathbf{n}$ is the unit vector in the magnetic field direction ( $\mathbf{n} \equiv \mathbf{H} / H$ ). As for now, we also consider both bulk (isotropic) and anisotropic (TB's) pinning forces. Also, for the former, we assume, as in [14], that $\mathbf{f}_{p}^{i}=-\gamma_{i}(v) \mathbf{v}$, where $\gamma_{i}(v)>0$ is the nonlinear phenomenological viscosity, which depends only on the magnitude of $v \equiv|\mathbf{v}|$. The anisotropic pinning force $\mathbf{f}_{p}^{a}$, which acts on the vortices at TB's, can be written as

$$
\mathbf{f}_{p}^{a}=-\gamma_{i}(v) \mathbf{v}-\gamma_{t}\left(\left|\mathbf{v}_{t}\right|\right) \mathbf{v}_{t}-\gamma_{l}\left(\left|\mathbf{v}_{l}\right|\right) \mathbf{v}_{l},
$$

where $\gamma_{t}$ and $\gamma_{l}$ are the average phenomenological transverse and longitudinal vortex pinning viscosities, respectively. Equations (1)-(3) allow us to write the force balance equation for the $k$-th vortex in much the same way as in [14]. Then, on averaging it over disorder, thermal fluctuations, and also vortex twin and bulk positions [14], we arrive at

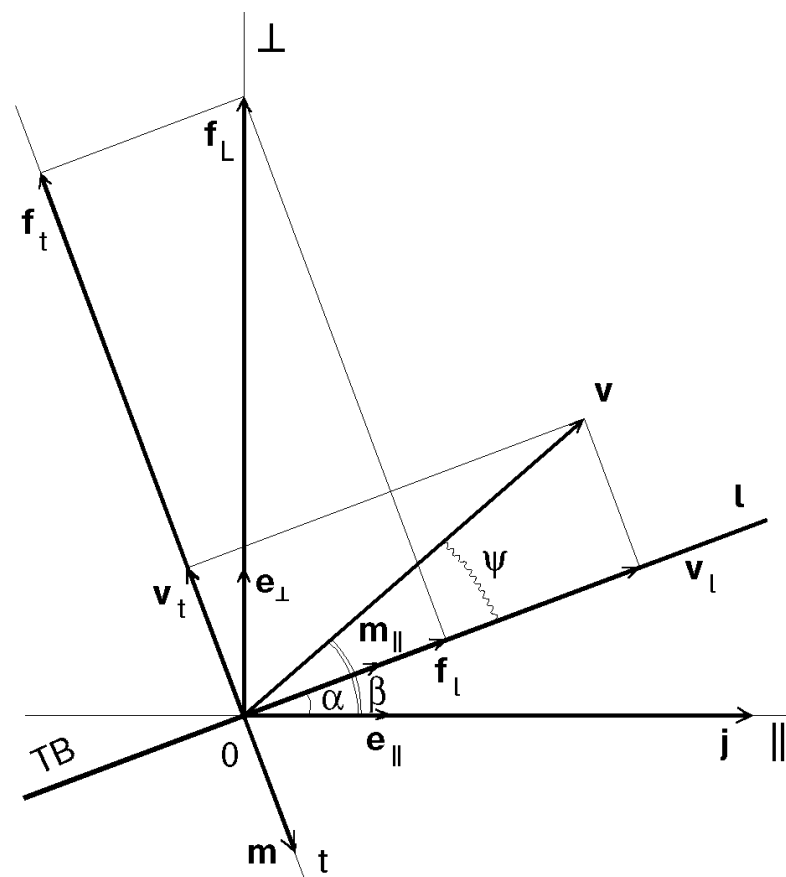

Fig. 2. The $a b$-plane geometry of $\mathbf{v}$ and $\mathbf{f}$ components in two coordinate systems: $(l, t)$ coordinates with unit vectors $\mathbf{m}_{\|}$ (along $\mathbf{j}$ ) and $\mathbf{m} ;(\perp, \|)$ coordinates with unit vectors $\mathbf{e}_{\|}$(along j) and $\mathbf{e}_{\perp} ; \mathbf{v}-$ average vortex velocity, $\mathbf{f}_{L}-$ Lorentz force, $\mathbf{j}-$ current density. Angles $\alpha, \beta, \psi-$ see text. the following dynamic equation for the average velocity of «effective» vortex in the crystal with unidirected TB's

$$
\begin{gathered}
\hat{\eta}_{i} \mathbf{v}+\alpha_{i} \mathbf{v} \times \mathbf{n}+ \\
+\varepsilon\left[\hat{\eta}_{t} \mathbf{v}_{t}+\alpha_{t} \mathbf{v}_{t} \times \mathbf{n}+\hat{\eta}_{l} \mathbf{v}_{l}+\alpha_{l} \mathbf{v}_{l} \times \mathbf{n}\right]=\mathbf{f}_{L},(4) \\
\hat{\eta}_{i} \equiv \eta_{i}+\gamma_{i}(v), \hat{\eta}_{t} \equiv \eta_{t}+\gamma_{t}\left(\left|\mathbf{v}_{t}\right|\right), \hat{\eta}_{l} \equiv \eta_{l}+\gamma_{l}\left(\left|\mathbf{v}_{l}\right|\right),
\end{gathered}
$$

where $\mathbf{f}_{L}=\left(\Phi_{0} / c\right) \mathbf{j} \times \mathbf{n}$ is the Lorenz force $\left(\Phi_{0}\right.$ is the flux quantum and $c$ is the velocity of light). Let $\mathbf{m}$ and $\mathbf{m}_{\|} \equiv \mathbf{z} \times \mathbf{m}$ be the unit vectors of $(t, l)$ coordinate system (see Fig. 2), directed perpendicularly and parallel to the TB's, and $\mathbf{z}$ be the unit vector along the $\mathbf{z}$-axis, which is perpendicular to the sample plane $(\mathbf{n}=n \mathbf{z}$, where $n= \pm 1)$. Then, taking into account that $\mathbf{v}=(c / H) \mathbf{E} \times \mathbf{n}$, where $\mathbf{E}$ is the in-plane electric field, we can arrive at a nonlinear Ohm's law in the form

$$
\begin{gathered}
\mathbf{j}=\left(c^{2} / H \Phi_{0}\right)\left\{\hat{\eta}_{i} \mathbf{E}+\alpha_{i} \mathbf{E} \times \mathbf{n}+\right. \\
\left.+\varepsilon\left[\hat{\eta}_{t} \mathbf{E}_{l}+\alpha_{t} \mathbf{E}_{l} \times \mathbf{n}+\hat{\eta}_{l} \mathbf{E}_{t}+\alpha_{l} \mathbf{E}_{t} \times \mathbf{n}\right]\right\} .
\end{gathered}
$$

Here $\mathbf{E}_{t}=E_{t} \mathbf{m}, \mathbf{E}_{l}=E_{l} \mathbf{m}_{\|}$and $E_{t}=\mathbf{m} \cdot \mathbf{E}, E_{l}=$ $=\mathbf{m}_{\|} \cdot \mathbf{E}$. Because of $E_{l}=(n H / c) v_{t}$ and $E_{t}=$ $=-(n H / c) v_{l}$, viscosities (5), actually, depend on corresponding electric-field components. form

Vector Eq. (6) can be represented in the scalar

$$
\left\{\begin{array}{l}
\hat{\sigma}_{l} E_{t}-\sigma_{H t} n E_{l}=j_{t} \\
\sigma_{H l} n E_{t}+\hat{\sigma}_{t} E_{l}=j_{l}
\end{array},\right.
$$

where $j_{t} \equiv \mathbf{m} \cdot \mathbf{j}, j_{l} \equiv \mathbf{m}_{\|} \cdot \mathbf{j}$, and the quantities $\hat{\sigma}_{l} \equiv\left(c^{2} / H \Phi_{0}\right)\left[\hat{\eta}_{i}(E)+\varepsilon \hat{\eta}_{l}\left(\left|E_{t}\right|\right)\right] \equiv \sigma_{i}(E)+\sigma_{l}\left(\left|E_{t}\right|\right)$,

$$
\hat{\sigma}_{t} \equiv\left(c^{2} / H \Phi_{0}\right)\left[\hat{\eta}_{i}(E)+\varepsilon \hat{\eta}_{t}\left(\left|E_{l}\right|\right)\right] \equiv \sigma_{i}(E)+\sigma_{t}\left(\left|E_{l}\right|\right)
$$

$$
\begin{gathered}
\sigma_{H t} \equiv-\left(\alpha_{i}+\varepsilon \alpha_{t}\right)\left(c^{2} / H \Phi_{0}\right) \\
\sigma_{H l} \equiv-\left(\alpha_{i}+\varepsilon \alpha_{l}\right)\left(c^{2} / H \Phi_{0}\right)
\end{gathered}
$$

are the additive functions of the appropriate viscosities and have a physical meaning of the corresponding components of the conductivity tensor (in $t-l$ representation):

$$
\hat{\sigma} \equiv\left(\begin{array}{cc}
\hat{\sigma}_{l} & -n \sigma_{H t} \\
n \sigma_{H l} & \hat{\sigma}_{t}
\end{array}\right) .
$$


The formal solution of Eqs. (7) as linear equations (but with nonlinear coefficients!) allows one to deduce the nonlinear Ohm's in the $\mathbf{E}(\mathbf{j})$ form (see also [16]):

$$
\begin{gathered}
\mathbf{E}=\hat{\rho}_{l} \mathbf{m}(\mathbf{m} \cdot \mathbf{j})+\hat{\rho}_{t} \mathbf{m}_{\|}\left(\mathbf{m}_{\|} \cdot \mathbf{j}\right)+ \\
+n\left[\hat{\rho}_{H l} \mathbf{m}_{\|}(\mathbf{m} \cdot \mathbf{j})-\hat{\rho}_{H t} \mathbf{m}\left(\mathbf{m}_{\|} \cdot \mathbf{j}\right)\right], \\
\hat{\rho_{l}} \equiv \hat{\sigma} / D_{\sigma}, \quad \hat{\rho}_{t} \equiv \hat{\sigma}_{l} / D_{\sigma}, \\
\hat{\rho}_{H l} \equiv-\hat{\sigma}_{H l} / D_{\sigma}, \quad \hat{\rho}_{H t} \equiv-\hat{\sigma}_{H t} / D_{\sigma},
\end{gathered}
$$

where $D_{\sigma} \equiv \hat{\sigma}_{l} \hat{\sigma}_{t}+\sigma_{H l} \sigma_{H t}$, and Eqs. (11) give the elements of resistivity tensor $\hat{\rho}$ which is the reciprocal of $\hat{\sigma}$. Although Eq. (10) formally resembles a similar expression for the linear Ohm's law in [13], its physical meaning is wider, because, generally, the resistivities $\hat{\rho}_{l}, \hat{\rho}_{t}, \hat{\rho}_{H l}, \hat{\rho}_{H t}$, are the nonlinear functions of $\mathbf{E}$ [see Eqs. (8)], and this circumstance is denoted by the superscript ${ }^{\wedge}{ }^{\wedge} »$. If E-dependence of $\hat{\rho}$ is irrelevant, then Eq. (10) is equivalent to the similar equation in [13] (see also [17]).

In experiment, one usually measures the longitudinal $E_{\|}$and transverse $E_{\perp}$ (with respect to j-direction) components of $\mathbf{E}$. In these $(\perp, \|)$ coordinates (see Fig. 2) the unit vectors are $\mathbf{e}_{\|} \equiv \mathbf{j} / j$, $\mathbf{e}_{\perp} \equiv \mathbf{z} \times \mathbf{j} / j$, and $\mathbf{E}=E_{\|} \mathbf{e}_{\|}+E_{\perp} \mathbf{e}_{\perp}$. Then there are simple relations between $E_{t}, E_{l}$ and $E_{\|}, E_{\perp}$ of the form

$$
\left\{\begin{array}{l}
E_{t}=x E_{\|}-y E_{\perp} \\
E_{l}=y E_{\|}+x E_{\perp}
\end{array} ; \quad\left\{\begin{array}{l}
E_{\|}=y E_{l}+x E_{t} \\
E_{\perp}=x E_{l}-y E_{t}
\end{array},\right.\right.
$$

where $x \equiv \mathbf{m} \cdot \mathbf{e}_{\|}$and $y \equiv \mathbf{m}_{\|} \cdot \mathbf{e}_{\|}$. Then, in view of (12), we have

$$
\begin{gathered}
E_{\|}=\left(\hat{\rho}_{\|}^{+}+n \hat{\rho_{\|}^{-}}\right) j, \quad E_{\perp}=\left(\hat{\rho_{\perp}^{+}}+n \hat{\rho}_{\perp}^{-}\right) j ; \\
\left\{\begin{array}{l}
\hat{\rho}_{\|}^{+} \equiv x^{2} \hat{\rho}_{l}+y^{2} \hat{\rho}_{t} \\
\hat{\rho}_{\perp}^{+} \equiv x y\left(\hat{\rho}_{t}-\hat{\rho}_{l}\right)
\end{array} ;\left\{\begin{array}{l}
\hat{\rho}_{\perp}^{-} \equiv x^{2} \hat{\rho}_{H l}+y^{2} \hat{\rho}_{H t} \\
\hat{\rho}_{\|}^{-} \equiv x y\left(\hat{\rho}_{H l}-\hat{\rho}_{H t}\right)
\end{array}\right.\right.
\end{gathered}
$$

Note, that the experimentally measured $\hat{\rho}_{\perp, \|}^{ \pm}$values, generally depend, as defined by Eqs. (14), on the angle $\alpha$ between $\mathbf{m}_{\|}$and $\mathbf{j}$ (see Fig. 2) in two ways. The explicit dependence on $\alpha$ can easily be seen from Eqs. (14), provided that the elements of the tensor $\hat{\rho}$ do not depend on $\mathbf{E}$, i.e., if $\hat{\rho} \rightarrow \rho$, where $\rho$ is the tensor of the linear Ohm's law. However, in nonlinear regimes there appears an additional nonlinear angular dependence of $\hat{\rho}_{\perp, \|}^{ \pm}$through the implicit dependence of $\hat{\rho}$-tensor elements on $E_{l}, E_{t}$ fields, which, in their turn, depend on the $\alpha$ value through $j_{t}=x j$ and $j_{l}=y j$ by Eqs. (7). Below we pay a special attention to this $\alpha$-dependence due to its nontriviality.

b) Scaling and «reconstruction» of $\hat{\sigma}$ in the a-pinning model. Equations (14) show that, in the case of linear Ohm's law, resistivities $\rho_{\|, \perp}^{ \pm}(\alpha)$ can be found for the sample with an arbitrary $\alpha$ value (see Fig. 1,a), if four current-independent intrinsic resistivities $\rho_{l}, \rho_{t}, \rho_{H l}, \rho_{H t}$ are known. In their turn, they can be measured experimentally ( $\ll$ reconstructed») in two special («reduced») geometries of experiment (see Figs. $1, b, 1, c$ ), namely, $\rho_{l}, \rho_{H l}-$ in the longitudinal L-geometry ( $\mathbf{j} \perp$ TB's), and $\rho_{t}$, $\rho_{H t}-$ in the transverse T-geometry ( $\mathbf{j} \|$ TB's). In these reduced LT-geometries the sample with unidirected twins behaves isotropically, because, by virtue of Eqs. (14), the two new resistivity components $\rho_{\perp}^{+}$and $\rho_{\|}^{-}$, which are specific to anisotropic geometry of general type $(\alpha \neq 0, \pi / 2)$, are equal to zero. Below we show that for the case of purely anisotropic pinning [ $a$-pinning model, $\gamma_{i}(v)=0$ ], the above-mentioned situation can be generalized to the nonlinear regime.

Actually, it can be shown that Eqs. (7) can be written for both L-geometry $\left(x \equiv x_{L}=1\right.$, $\left.y \equiv y_{L}=0\right)$ and T-geometry $\left(x_{T}=0, y_{T}=1\right)$ as $\left\{\begin{array}{l}\hat{\sigma}_{l}\left(E_{\|}^{L}\right) E_{\|}^{L}-\sigma_{H t} E_{\perp}^{L}=j \\ \hat{\sigma}_{t}\left(E_{\perp}^{L}\right) E_{\perp}^{L}+\sigma_{H l} E_{\|}^{L}=0\end{array},\left\{\begin{array}{l}\hat{\sigma}_{l}\left(E_{\perp}^{T}\right) E_{\perp}^{T}+\sigma_{H t} E_{\|}^{T}=0 \\ \hat{\sigma}_{t}\left(E_{\|}^{T}\right) E_{\|}^{T}-\sigma_{H l} E_{\perp}^{T}=j\end{array}\right.\right.$

Now we assume that four CVC's, namely $E_{\|}^{L}(j)$, $E_{\perp}^{L}(j), E_{\|}^{T}(j), E_{\perp}^{T}(j)$, are experimentally known. Let also $E_{\|}^{L}=f_{L}\left(E_{\perp}^{L}\right)$ and $E_{\|}^{T}=f_{T}\left(E_{\perp}^{T}\right)$, where the functions $f_{L}(x)$ and $f_{T}(x)$ are known, too. Then, after some algebra with Eqs. (15) we arrive at

$$
\begin{gathered}
\left\{\begin{array}{l}
\sigma_{H t}=-j /\left[E_{\perp}^{L}+f_{T}\left(E_{\|}^{L}\right)\right] \\
\sigma_{H l}=-j /\left[E_{\perp}^{T}+f_{L}\left(E_{\|}^{T}\right)\right]
\end{array}\right. \\
\left\{\begin{array}{l}
\hat{\sigma}_{l}(x)=\left[j_{\|}^{L}(x) / x\right] /\left[1+f_{L}^{-1}(x) / f_{T}(x)\right] \\
\hat{\sigma}_{t}(x)=\left[j_{\|}^{T}(x) / x\right] /\left[1+f_{T}^{-1}(x) / f_{L}(x)\right]
\end{array},\right.
\end{gathered}
$$

where $j_{\|}^{L}\left(E_{\|}^{L}\right), j_{\|}^{T}\left(E_{\|}^{T}\right)$ are the functions inverted to the functions $E_{\|}^{L}(j), E_{\|}^{T}(j)$, respectively. Equations (16) give the complete and exact solution of the $\hat{\sigma}$-tensor reconstruction problem in the $a$-pinning model. They allow us to express exactly the longi- 
tudinal and transverse CVC's of the sample with arbitrary angle $\alpha$ (see Fig. 1,a) in terms of four CVC's experimentally measured in LT-geometries [17] at arbitrary constant values of $\sigma_{H l}, \sigma_{H t}$; the expressions for the last are, in fact, the desired scaling relations in the $a$-pinning model. If, as is often the case in experiment [5,9], the Hall components of the $\hat{\sigma}$-tensor are considerably smaller then the diagonal components, then Eqs. (16) are greatly simplified to

$$
\left\{\begin{array}{l}
\sigma_{H t} \approx-j / f_{T}\left[E_{\|}^{L}(j)\right] \\
\sigma_{H l} \approx-j / f_{L}\left[E_{\|}^{T}(j)\right]
\end{array},\left\{\begin{array}{l}
\hat{\sigma}_{l}(x) \approx j_{\|}^{L}(x) / x \\
\hat{\sigma}_{t}(x) \approx j_{\|}^{T}(x) / x
\end{array} .\right.\right.
$$

Physical realization of pure anisotropic pinning considered here is most probable in the temperature range $T_{\mathrm{dp}}^{i}<T<T_{\mathrm{dp}}^{\|}$, where $T_{\mathrm{dp}}^{i}$ and $T_{\mathrm{dp}}^{\|}$are depinning temperatures [1] for the point pins and the longitudinal motion of vortices on TB's, respectively.

c) Guiding analysis. Below we present the main results of studying the GM in the $a$-pinning model. For simplicity, we here neglect generally small nondiagonal Hall terms in the $\boldsymbol{\sigma}$-tensor (Eq. (13)).

First we consider the linear case, where $\rho_{l}=\rho_{\|}^{+L}, \rho_{t}=\rho_{\|}^{+T}$, i.e., $\rho_{l}, \rho_{t}$ values can be measured in LT-geometries. At arbitrary angle $\alpha \neq 0, \pi / 2$, the directions of $\mathbf{v}$ and $\mathbf{f}$ do not coincide (see Fig. 2), if $\eta_{l} \neq \eta_{t}$ (i.e., $\rho_{t} \neq \rho_{l}$ ). Let us define the auxiliary angle $\psi$ as a measure of competition between guided $\left(\sim v_{l}\right)$ and transverse $\left(\sim v_{t}\right)$ motion of vortices across TB's («slipping», in terms of [12]). It follows from Eq. (4) that

$$
\tan \psi \equiv v_{t} / v_{l}=\tan \alpha_{c} / \tan \alpha,
$$

where $\tan \alpha_{c} \equiv \rho_{t} / \rho_{l}$. In general, $0<\alpha_{c}<\pi / 2$, however, normally $[2,8] \rho_{t}<\rho_{l}$ and $\alpha_{c}<\pi / 4$. We also introduce another angle $\beta \equiv \alpha+\psi$ (see Fig. 2), which can be measured experimentally [12], because

$$
\cot \beta \equiv-E_{\perp}^{+} / E_{\|}^{+}=-\rho_{\perp}^{+}(\alpha) / \rho_{\|}^{+}(\alpha) .
$$

As it follows from (19) and the definition of $\beta$,

$$
\cot \beta=\tan \alpha\left(1-\tan \alpha_{c}\right) /\left(\tan ^{2} \alpha+\tan \alpha_{c}\right) .
$$

From Eq. (20) one can deduce that the $\beta(\alpha)$ dependence is always nonmonotonic (see Fig. 3 ). The extreme value of $\beta_{\text {ext }}\left(\beta_{\max }\right.$ at $\alpha_{c}>\pi / 4$ or $\beta_{\min }$ at $\left.\alpha_{c}<\pi / 4\right)$ is attained at the $\tan \alpha_{0}=\left(\tan \alpha_{c}\right)^{1 / 2}$ and $\beta_{\text {ext }}=2 \alpha_{0}$. Experimentally, of special interest

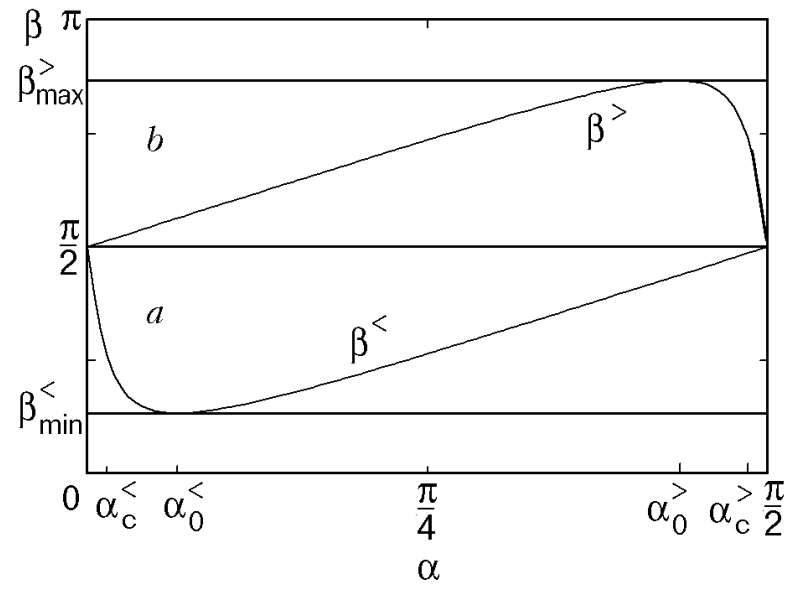

Fig. 3. Schematic nonmonotonic dependence of $\beta$ on $\alpha$, where $\beta$ is the angle between $\mathbf{j}$ and $\mathbf{v}$ (see Fig. 2) and $\alpha$ is the angle between $\mathbf{j}$ and $\mathbf{m}_{\|}: \alpha_{c}=\alpha_{c}^{<}, \beta=\beta^{<}(a) ; \alpha_{c}=\alpha_{c}^{>}, \beta=\beta^{>}(b)$.

are the cases where $\cot \beta>>1$, i.e., the transverse electric field $E_{\perp}^{+}$is considerably greater than the longitudinal field $E_{\|}^{+}$due to the dominant role of the GM. More detailed examination of Eq. (20) shows that the most favorable conditions for that case will be at $\tan \alpha_{c}<<1$ and $\tan \alpha<<1$. In fact, there are two limiting cases, were $\left|E_{\perp}^{+}\right|>>\left|E_{\|}^{+}\right|$, namely

$\cot \beta \approx\left\{\begin{array}{l}\frac{\tan \alpha}{\tan \alpha_{c}}>>1, \tan ^{2} \alpha<\tan \alpha_{c}<<\tan \alpha \\ \frac{1}{\tan \alpha}>>1, \tan \alpha_{c}<<\tan ^{2} \alpha\end{array}\right.$

The situation is quite real experimentally, because in experiment [8] it was shown that $\tan \alpha_{c}<10^{-6}$ at $T \approx 87 \mathrm{~K}$ for YBCO (see Fig. 1, curves 1 and 5 in [8]). Note also that the distinction between cases a) and b) in Eq. (21) follows from the fact that the angle $\beta$ always shows an extreme behavior in the vicinity of angle $\alpha_{0}$ (see Fig. 3).

The linear result can be generalized to the nonlinear regimes if we replace $\tan \alpha_{c}$ by $\tan \hat{\alpha}_{c} \equiv$ $\equiv \hat{\rho}_{t}\left(\left|E_{l}\right|\right) / \hat{\rho}_{l}\left(\left|E_{t}\right|\right)$, i.e., we take into account that $\alpha_{c} \rightarrow \alpha_{c}(\alpha, j)$. It can be demonstrated (see Eqs. (8) and (11)) that

$$
\tan \hat{\alpha}_{c}=\rho_{T}\left(j_{l}\right) / \rho_{L}\left(j_{t}\right)=\left[\frac{E_{\|}^{T}(j \cos \alpha)}{E_{\|}^{L}(j \sin \alpha)}\right] \tan \alpha,
$$

where, as previously, $E_{\|}^{L}(j)=j \rho_{L}(j)$ and $E_{\|}^{T}(j)=$ $=j \rho_{T}(j)$ are longitudinal CVC's in LT-geometries, respectively. Because $\hat{\alpha}_{c}=\hat{\alpha}_{c}(\alpha, j)$, the nonlinear 
$\alpha-j$ dynamics of $\cot \hat{\beta}$ may be more complicated than in the linear case. The two limits $(\alpha \rightarrow 0$ and $\alpha \rightarrow \pi / 2)$ are of particular interest. For example, if $\alpha \rightarrow \pi / 2$, then in the creep regime (for the power-law CVC's) we may have $\rho_{T}(j \cos \alpha) \rightarrow$ $\rightarrow \rho_{T}[j(\pi / 2-\alpha)] \ll \rho_{T}(j)$, whereas $\rho_{L}(j \sin \alpha) \approx$ $\approx \rho_{L}(j)$. So, if for LT-geometries at fixed $j$ the values of $\rho_{T}(j)$ and $\rho_{L}(j)$ are of the same order, i.e., $\tan \hat{\alpha}_{c} \leqslant 1$ for $\alpha \approx \pi / 4$ (weak guiding), then in the limit $\alpha \rightarrow \pi / 2$ there should be $\tan \hat{\alpha}_{c}<<1$, i.e., we can expect an essential nonlinear enhancement of the guiding effect. Similar reasoning for $\alpha \rightarrow 0$ shows that $\tan \hat{\alpha}_{c}>>1$ is feasible; then it is possible that in the process of $\alpha$ increase from 0 to $\pi / 2$ at fixed $j$ the observed voltage $V_{\perp}^{+}$changes its sign, passing over $V_{\perp}^{+}=0$ at $\tan \alpha_{c}=1$. Yet, from the experimental viewpoint, it is of greater interest to realize this transition for the given sample ( $\alpha=$ const) by changing the current; qualitative analysis of these effects for the power-law CVC has been made [17].

Now we show that both longitudinal $E_{\|}^{\alpha}(j)$ and transverse $E_{\perp}^{\alpha}(j)$ CVC's of the sample with an arbitrary $\alpha$ value in the model under study can be expressed through the longitudinal CVC's in LT-geometries. Actually, in the $a$-pinning model $E_{t}=E_{t}\left(j_{t}\right)$ and $E_{l}=E_{l}\left(j_{l}\right)$ (see Eqs. (7), where small Hall terms are ignored). Then it follows from Eqs. (12), if we apply them to the LT-geometries, that $E_{t}\left(j_{t}\right)=E_{\|}^{L}\left(j_{t}\right)$ and $E_{l}\left(j_{l}\right)=E_{\|}^{T}\left(j_{l}\right)$. The repeated use of Eqs. (12) yields

$$
\left\{\begin{array}{l}
E_{\|}^{\alpha}(j)=x E_{\|}^{L}(x j)+y E_{\|}^{T}(y j) \\
E_{\perp}^{\alpha}(j)=x E_{\|}^{T}(y j)-y E_{\|}^{L}(x j)
\end{array} .\right.
$$

Note the nontrivial angular dependence in the arguments of $E_{\|}^{L}$ and $E_{\|}^{T}$ functions. Equatious (23) also show how the peculiarities of CVC's in the «basic» LT-geometries are manifested in the $E_{\|, \perp}^{\alpha}(j)$ CVC's. First we shall study the $\alpha$-dependence of critical current densities $j_{c}(\alpha)$ in terms of the basic critical current densities of our model $j_{c}^{L} \equiv j_{c}(\pi / 2)$ and $j_{c}^{T} \equiv j_{c}(0)$ in LT-geometries, respectively. For the analysis we assume the simplest form of CVC's in LT-geometries

$$
\begin{aligned}
& E_{\|}^{L}(j)=\rho_{l}\left(j-j_{c}^{L}\right) \theta\left(j-j_{c}^{L}\right) ; \\
& E_{\|}^{T}(j)=\rho_{t}\left(j-j_{c}^{T}\right) \theta\left(j-j_{c}^{T}\right),
\end{aligned}
$$

where $\theta(x)=1$ for $x>0$ and $\theta(x)=0$ for $x<0$. Using these «ideal» CVC's we ignore the creep and, at the same time, we fix the singularities of CVC's in the form of a kink at $j=j_{c}$. Substitution of Eqs. (24) into Eqs. (23) gives the analytical form of CVC's.

But it is more instructive to analyse these equations on the $\left(j_{t}, j_{l}\right)$ plane. In Fig. 4 the first quadrant of the plane is divided by straight lines $j_{l}=j_{c}^{T}$ and $j_{t}=j_{c}^{L}$ into four regions. The end of the vector $\mathbf{j}$ with the coordinates $(j \sin \alpha, j \cos \alpha)$ dependent on $j, \alpha$ values, can belong to each of these regions with different physical meaning of dynamic states of the vortex system.

The region of «full pinning» (FP in Fig. 4), where $E_{\|}^{\alpha}(j)=E_{\perp}^{\alpha}(j)=0$, is shown by the unshaded rectangle and its diagonal determines the critical angle $\alpha^{*}\left(\tan \alpha^{*} \equiv j_{c}^{L} / j_{c}^{T}\right)$. Then it is easy to see that

$$
j_{c}(\alpha) \equiv\left\{\begin{array}{ll}
j_{c}^{>}(\alpha)=j_{c}^{L} / \sin \alpha & \alpha>\alpha^{*} \\
j_{c}^{<}(\alpha)=j_{c}^{T} / \cos \alpha & \alpha<\alpha^{*}
\end{array} .\right.
$$

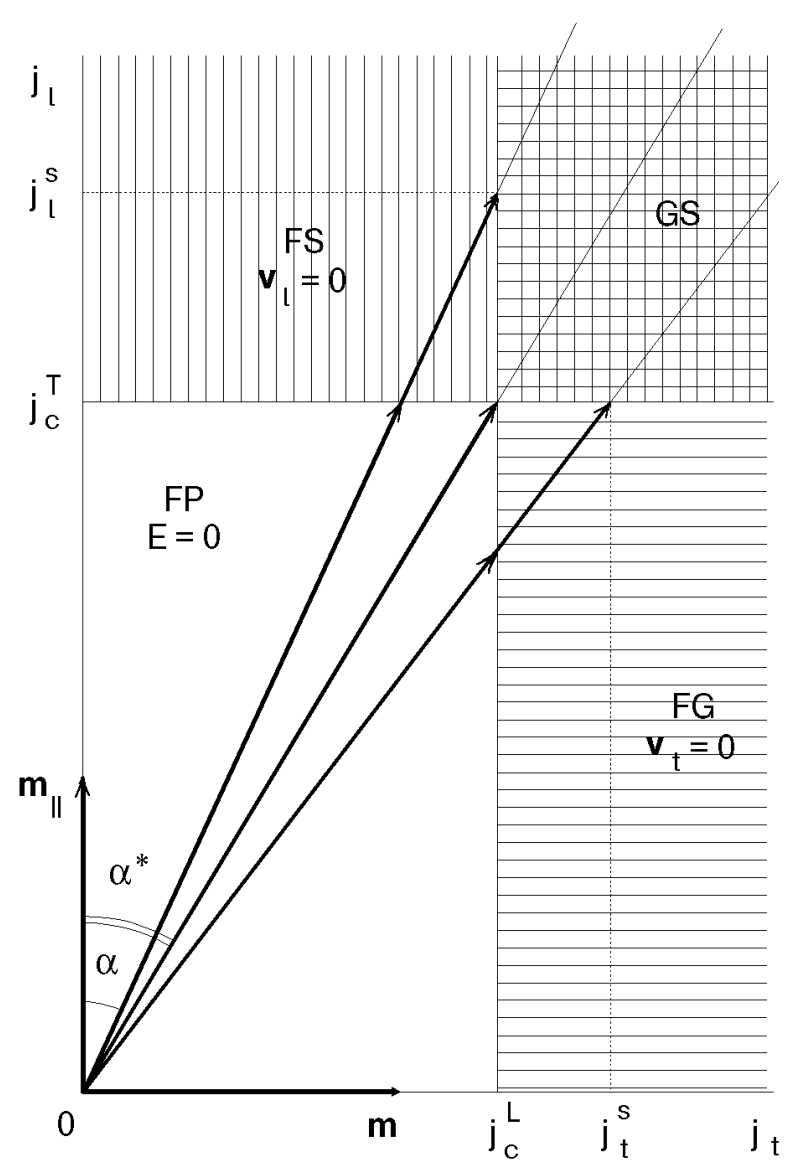

Fig. 4. Schematic diagram of the dynamic states of the vortex system on $\left(j_{l}, j_{t}\right)$ plane. There are four regions: FP - full pinning, FS - full slipping, FG - full guiding, GS - guiding and slipping. $j_{c}^{L}$ and $j_{c}^{T}-$ critical current densities in L- and T-geometries, respectively. 
In the region of «full slipping» (FS in Fig. 4), shaded by vertical lines, $\alpha<\alpha^{*}, j_{t}(\alpha)<j_{c}^{L}$, and the vortices are moving normally to the TB's, i.e., $\mathbf{v}_{F S}=\mathbf{v}_{t} \| \mathbf{m}$. The «full guiding» (FG) region is shaded in Fig. 4 by horizontal lines, it represents the fully GM of vortices with $\mathbf{v}_{F G}=\mathbf{v}_{l} \| \mathbf{m}_{\|}$, because at $\alpha>\alpha^{*}$ we always have $j_{l}(\alpha)<j_{c}^{T}$. And lastly, the «slipping and guiding» (SG) region, shaded by crossed lines, realizes the coexistence of slipping and guiding, where $\mathbf{v}=\mathbf{v}_{t}+\mathbf{v}_{l}$ with $\mathbf{v}_{t} \neq 0$ and $\mathbf{v}_{l} \neq 0$.

If, for the given sample with fixed $\alpha=\alpha_{j}$, the transport current is increasing from zero, then, depending on the $\alpha, j$ values it is possible to realize sequentially different variants of intersection by the end of $\mathbf{j}$-vector of the boundaries between the neighboring regions (see Fig. 4). For example, if $\alpha>\alpha^{*}$, then the series of intersections $\mathrm{FP} \rightarrow \mathrm{FG} \rightarrow \mathrm{GS}$ exists. Since a new source of dissipation appears, at each of the intersection, the longitudinal CVC of the sample $E_{\|}^{\alpha}(j, \alpha)$ acquires a kink (inflection point) at corresponding values of $j$. In general, there are two such kinks on the CVC (if $\left.\alpha \neq \alpha^{*}, 0, \pi / 2\right)$; only in the case $\alpha=\alpha^{*}$ these two kinks merge into one.

In conclusion, we note, that anisotropic transport effects caused by unidirected twins have only begun to be observed experimentally; until now, all the measurements were taken on YBCO single crystals [8-10]. However, recently fabricated [18] caxis-oriented YBCO thin films with unidirected twins, owing to a more pronounced anisotropy of their resistive properties and attainability of higher current densities without overheating, might appear more suitable for observation of the predicted here nonlinear effects than the crystals.
I am grateful to A. K. Soroka and A. A. Soroka for their help in preparing this paper for publication.

1. G. Blatter, M. V. Feigelman, V. B. Geshkenbein, A. I. Larkin, and V. M. Vinokur, Rev. Mod. Phys. 66, 1125 (1994).

2. S. Fleshler, W.-K. Kwok, U. Welp, V. M. Vinokur, M. K. Smith, J. Downey, and G. W. Crabtree, Phys. Rev. B47, 14448 (1993).

3. G. W. Crabtree, G. K. Leaf, H. G. Kaper, V. M. Vinokur, A. E. Koshelev, D. W. Braun, D. M. Levine, W. K. Kwok, and J. A. Fendrich, Physica C263, 401 (1996).

4. J. Groth, C. Reichhardt, C. J. Olson, S. B. Field, and F. Nori, Phys. Rev. Lett. 77, 3625 (1996).

5. T. R. Chien, T. W. Jing, N. P. Ong, and Z. Z. Wang, Phys. Rev. Lett. 66, 3075 (1991).

6. T. R. Chien, D. A. Brawner, Z. Z. Wang, and N. P. Ong, Phys. Rev. B43, 6242 (1991).

7. A. V. Samoilov, A. Legris, F. Rullier-Albenque, P. Lejay, S. Bouffard, Z. G. Ivanov, and L.-G. Johansson, Phys. Rev. Lett. 74, 2351 (1995).

8. A. V. Bondarenko, M. A. Obolenskii, R. V. Vovk, A. A. Prodan, V. A. Shklovskij, A. G. Sivakov, in: Proceedings of the 7th IWCC in Superconductors, Alpbach, Austria, 1994, H. W. Weber (ed.), World Scientific, Singapore (1994), p. 177.

9. A. A. Prodan, V. A. Shklovskij, V. V. Chabanenko, A. V. Bondarenko, M. A. Obolenskii, H. Szymczak, and S. Piechota, Physica C302, 271 (1998).

10. H. Ghamlouch and M. Aubin, Physica C269, 163 (1996).

11. Y. Mawatari, Phys. Rev. B56, 3433 (1997).

12. A. K. Niessen and C. H. Weijsenfeld, J. Appl. Phys. 40, 384 (1969).

13. E. B. Sonin and A. L. Kholkin, Fiz. Tverd. Tela 34, 1147 (1992) [Sov. Phys. Solid State 34, 610 (1992)].

14. V. M. Vinokur, V. B. Geshkenbein, M. V. Feigelman, and G. Blatter, Phys. Rev. Lett. 71, 1242 (1993)].

15. Wu Liu, T. W. Clinton, and C. J. Lobb, Phys. Rev. B52, 7482 (1995).

16. V. A. Shklovskij, Fiz. Nizk. Temp. 23, 1134 (1997) [Low Temp. Phys. 23, 853 (1997)].

17. V. A. Shklovskij, submitted to Phys. Rev. B.

18. C. Villard, G. Koren, D. Cohen, E. Polturac, Phys. Rev. Lett. 77, 3913 (1996). 